\title{
Novel optical pulse characterization technique using sinusoidal optical phase modulation with a broad usable modulation amplitude range
}

\author{
Weihua Guo ${ }^{1}$, Douglas Reid ${ }^{2}$, John O'Dowd ${ }^{1}$, Liam Barry ${ }^{2}$, and John F. Donegan ${ }^{1}$ \\ 1. Semiconductor Photonics Group, School of Physic, Trinity College, Dublin 2, Ireland \\ 2. Radio and Optical Communications Laboratory, School of Electronic Engineering, Dublin City University, Dublin 9, Ireland
}

Advanced optical pulse characterization methods are essential for high-speed optical communications. Recently non-iterative characterization of general optical pulses using sinusoidal optical phase modulation has been reported [1-2]. However, the usable phase modulation amplitude with this method has a relatively small upper limit $(0.88$ radians [1]). In this paper we propose a novel non-iterative optical pulse characterization technique using sinusoidal optical phase modulation, which can work with modulation amplitude up to 1.9 radians. The larger usable modulation amplitude would make the proposed method more tolerable to noise present in the recorded pulse spectrum after undergoing phase modulation.

The spectral intensity of the pulse after undergoing the sinusoidal phase modulation can be expressed as

$$
Q_{k}=\sum_{m} J_{m}^{2}(A) P_{k-m}+2 \sum_{m, n(m) n)} J_{n}(A) J_{m}(A) \sqrt{P_{k-n} P_{k-m}} \cos \left(\left(\phi_{k-n}-\phi_{k-m}\right)-(m-n) \psi\right)
$$

where $A$ is the phase modulation amplitude, $\psi$ is the phase related to the delay between the pulse train and the modulation signal, $P_{k}$ and $\phi_{k}$ are the original pulse spectral intensity and phase. In the proposed method, the phase $\psi$ is varied and simultaneously the spectral intensity $Q_{k}$ is recorded and used to calculate the following harmonics ${ }_{q_{k, s}}=\frac{1}{2 \pi} \int_{0}^{2 \pi} Q_{k}(\psi) \exp (j s \psi) d \psi$. The $1^{\text {st }}$ harmonic gives

$$
q_{k, 1}=\sum_{m=-M}^{M} J_{m-1}(A) J_{m}(A) \sqrt{P_{k-m+1} P_{k-m}} \exp \left(j\left(\phi_{k-m+1}-\phi_{k-m}\right)\right)
$$

Equation (2) is a linear equation through which the spectral phase differentiation can be found which can then be integrated to find the spectral phase. The pulse spectral intensity can be measured directly so by this way the pulse can be fully characterized. $M$ is the termination order of the Bessel function, which is selected to be large enough to ensure that $J_{M}^{2}(A)$ is $60 \mathrm{~dB}$ lower than the peak of the sequence of $J_{m}^{2}(A)$.

The proposed scheme is checked first by simulating the characterization of three kinds of pulses all repeated at $10 \mathrm{GHz}$ : 2-ps Gaussian pulses, chirped $33 \% \mathrm{RZ}$ pulses and chirped $67 \% \mathrm{CS}-\mathrm{RZ}$ pulses [1-2]. We find that satisfactory pulse characterizations (Root-mean-square (RMS) of the field error $<0.1$ in Fig. 1(a)) can be obtained through the proposed method even when the phase modulation amplitudes is up to 1.9 radians as shown in Fig. 1 (a). The method is then validated experimentally by characterizing a 2-ps pulse train repeating at 10 $\mathrm{GHz}$, generated from a tunable mode locked laser (U2t TMLL1550). $\mathrm{A} \mathrm{LiNbO}_{3}$ phase modulator driven by the same $10 \mathrm{GHz}$ clock as is applied to the TMLL provides the phase modulation. The relative phase between the $\mathrm{RF}$ signal driving the TMLL and the phase modulator is shifted through a voltage controlled analogue RF phase shifter. An optical spectrum analyser (OSA) with a resolution bandwidth of $0.02 \mathrm{~nm}$ is used to record the phase modulated pulse spectra with a noise floor about $40 \mathrm{~dB}$ lower than the spectral peak. The relative phase is stepped uniformly over $2 \pi$ in 16 steps with a spectrum recorded for each of the 16 steps. The pulse spectra intensity is directly measured by the OSA with the phase modulation turned off. The modulation amplitude is determined to be 1.32 radians. The recovered pulse intensity and phase versus time are shown in Fig. 1 (b). Also shown is the result from the standard SHG-FROG technique. Very good agreenment can be seen from Fig. 1 (b).

(a)

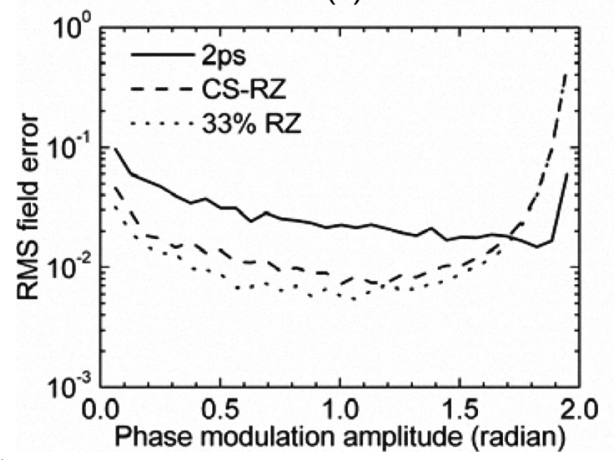

(b)

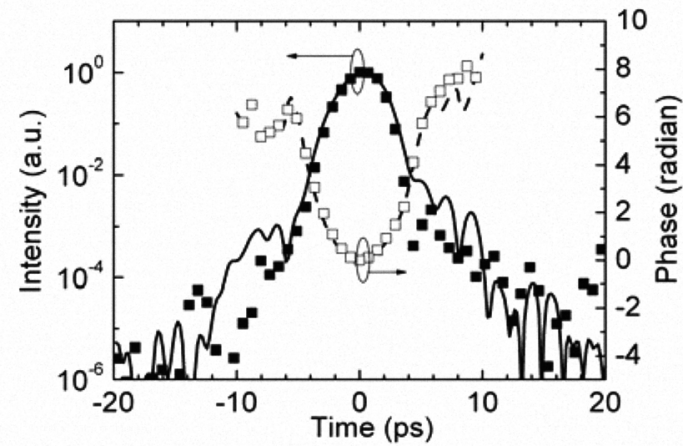

Fig. 1 (a) RMS field error versus the phase modulation amplitude for three kinds of pulses simulated; (b) Measured pulse intensity and phase. The solid (dashed) curve is the pulse intensity (phase) measured by the proposed method. The solid (hollow) squares are the pulse intensity (phase) measured by the SHG-FROG technique.

\section{References}

[1] I. Kang, and C. Dorrer, "Method of optical pulse characterization using sinusoidal optical phase modulations," Opt. Lett., 32, 2538 (2007). [2] I. Kang, and C. Dorrer, "Novel method of optical pulse characterization using sinusoidal optical phase modulation," CLEO 2006, Technical Digest, paper CTuC5. 